\title{
DUAL EXTREMUM PRINCIPLES FOR A CLASS OF INTERFACE PROBLEMS*
}

\author{
BY \\ D. THANGARAJ AND S. N. VENKATARANGAN \\ Indian Institute of Technologv, Madras
}

1. Introduction. Dual extremum principles [1] have been used to obtain error bounds for a wide class of boundary value problems.

In this note, the dual extremum principles are formulated associated with the set of operator equations of the form

$$
L_{j} \varphi_{j}=T_{j}^{*} k_{j} T_{j} \varphi_{j}=f_{j}\left(\varphi_{j}\right) \quad \text { in } D_{j}, \quad j=1,2,
$$

with the boundary conditions

$$
\varphi_{j}=\alpha_{j} \quad \text { on } \Gamma_{j}=\partial D_{j}-\Gamma, \quad j=1,2,
$$

and the interface matching conditions

$$
\begin{gathered}
\sigma_{1}\left(\varphi_{2}-\varphi_{1}-g\right)=0 \text { on } \Gamma, \\
\sigma_{1}^{*} k_{1} T_{1} \varphi_{1}-\sigma_{2}^{*} k_{2} T_{2} \varphi_{2}=h(x, y) \text { on } \Gamma .
\end{gathered}
$$

Hereafter, whenever the subscript " $j$ " appears, it should be understood that $j=1,2$ unless otherwise specified. $D_{j}$ is a closed convex region in $R^{n}, n=1,2,3$, with boundary $\partial D_{j}, \Gamma=\partial D_{1} \cap \partial D_{2}$ being the interface. $T_{j}^{*}: H_{j}^{u} \rightarrow H_{j}^{\varphi}$ on $D_{j}$ is the formal adjoint operator of $T_{j}: H_{j}^{\varphi} \rightarrow H_{j}^{u}$ on $D_{j}$ such that

$$
\left(u, T_{j} \varphi\right)_{j}=\left\langle T_{j}^{*} u, \varphi\right\rangle_{j}+\left(u, \sigma_{j} \varphi\right)_{\partial D_{j}} ;
$$

$\sigma_{j}^{*}: H_{j}^{u} \rightarrow H_{j}^{\varphi}$ on $\partial D_{j}$ is the adjoint operator of $\sigma_{j}: H_{j}^{\varphi} \rightarrow H_{j}^{u}$ such that

$$
\left(u, \sigma_{j} \varphi\right)=\left\langle\sigma_{j}^{*}, u, \varphi\right\rangle \text { on } \partial D_{j}
$$

and $f_{j}\left(\varphi_{j}\right): H_{j}^{\varphi} \rightarrow H_{j}^{\varphi} \cdot H_{j}^{\varphi}$ and $H_{j}^{u}$ are Hilbert spaces of functions defined on $D_{j}$ with the innerproducts $\langle,\rangle_{j}$ and $(,)_{j}$, respectively, and $\alpha_{j}$ and $g$ and $h$ are known functions defined on the boundary and the interface, respectively. These kinds of problems occur in many branches of mathematical physics such as heat conduction [2], electromagnetic theory [3] and fluid dynamics [4]. When $T_{j}=\operatorname{grad}, g=0$ and $k_{j}$ is the thermal conductivity of the medium $D_{j}$, the problem (1)-(4) reduces to finding the steady state temperature distribution $\varphi_{j}$ in the media which are in perfect contact, $h(x, y)$ being the source function

${ }^{*}$ Received July 25, 1984. 
defined on the interface [2]. The interface problem (1)-(4) reduces to finding the magnetic vector potential $\varphi_{j}$ in a medium such that the magnetic vector potential and the magnetic intensity at the interface are continuous when $T_{j}=$ curl and $k_{j}^{-1}$ is the magnetic permeability of the medium $D_{j}$. After deriving the bounding functionals to the extremizing functional, an error estimate is obtained for the approximate solution in terms of the bounding functionals. The theory is illustrated by the steady state heat conduction in a composite medium and the approximate solution is obtained by the method of Kantorovich [5].

2. Variational formulation. Consider the action functional

$$
\begin{aligned}
I\left(u_{1}, u_{2}, \varphi_{1}, \varphi_{2}\right)= & \sum_{j=1}^{2}\left[\left(u_{j}, T_{j} \varphi_{j}\right)_{j}-W_{j}\left(u_{j}, \varphi_{j}\right)\right. \\
& \left.+\left\langle\sigma_{j}^{*} u_{j}, \alpha_{j}-\varphi_{j}\right\rangle_{\Gamma}\right]+\left\langle\sigma_{1}^{*} u_{1}, \varphi_{2}-\varphi_{1}-g\right\rangle_{\Gamma}-\left\langle h, \varphi_{2}\right\rangle_{\Gamma} \\
= & \sum_{j=1}^{2}\left[\left\langle T_{j}^{*} u_{j}, \varphi_{j}\right\rangle_{j}-W_{j}\left(u_{j}, \varphi_{j}\right)+\left\langle\sigma_{j}^{*} u_{j}, \alpha_{j}\right\rangle_{\Gamma}\right] \\
& +\left\langle\varphi_{2}, \sigma_{1}^{*} u_{1}-\sigma_{2}^{*} u_{2}-h\right\rangle_{\Gamma}-\left\langle\sigma_{1}^{*} u_{1}, g\right\rangle_{\Gamma},
\end{aligned}
$$

where

$$
W_{j}\left(u_{j}, \varphi_{j}\right)=\frac{1}{2}\left(\frac{u_{j}}{k_{j}}, u_{j}\right)+\left\langle 1, F_{j}\left(\varphi_{j}\right)\right\rangle
$$

and

$$
F_{j}\left(\varphi_{j}\right)=\int^{\varphi_{j}} f_{j}(t) d t
$$

A necessary and sufficient condition for stationary behaviour of $I$ at $\left(u_{j}, \varphi_{j}\right)$ is that the Frechet derivatives

$$
\frac{\delta I}{\delta u_{j}}=\frac{\delta I}{\delta \varphi_{j}}=0
$$

This leads to

$$
\begin{gathered}
T_{j} \varphi_{j}=\frac{\delta W_{j}}{\delta u_{j}} \text { in } D_{j}, \\
\varphi_{j}=\alpha_{j} \text { on } \Gamma_{j}, \\
\sigma_{1}\left(\varphi_{2}-\varphi_{1}-g\right)=0 \text { on } \Gamma,
\end{gathered}
$$

and

$$
\begin{gathered}
T_{j}^{*} u_{j}=\frac{\delta W_{j}}{\delta \varphi_{j}} \text { in } D_{j}, \\
\sigma_{1}^{*} u_{1}-\sigma_{2}^{*} u_{2}=h \text { on } \Gamma .
\end{gathered}
$$


Equations (11)-(15) are nothing but the canonical forms of the interface problem described by equations (1)-(4). Hence it is evident that $\left(u_{j}, \varphi_{j}\right)$ is the solution of (1)-(4).

3. Dual extremum principles and error estimate. Let $\left(u_{j}, \varphi_{j}\right)$ be the exact solution of (1)-(4) so that

$$
\frac{\delta I}{\delta u_{j}}=\frac{\delta I}{\delta \varphi_{j}}=0 \quad \text { at }\left(u_{j}, \varphi_{j}\right) .
$$

Define two sets of functions

$$
\begin{aligned}
& S_{1}=\left\{\left(u_{j}, \varphi_{j}\right) / \frac{\delta I}{\delta u_{j}}=0\right\}, \\
& S_{2}=\left\{\left(u_{j}, \varphi_{j}\right) / \frac{\delta I}{\delta \varphi_{j}}=0\right\} .
\end{aligned}
$$

Using (7) and (8) form

$$
J\left(B_{1}, B_{2}\right)=I\left(u_{1}^{*}, u_{2}^{*}, B_{1}, B_{2}\right), \quad\left(u_{j}^{*}, B_{j}\right) \in S_{1},
$$

and

$$
G\left(A_{1}, A_{2}\right)=I\left(A_{1}, A_{2}, \varphi_{1}^{*}, \varphi_{2}^{*}\right), \quad\left(A_{j}, \varphi_{j}^{*}\right) \in S_{2},
$$

where $u_{j}^{*}=u_{j}^{*}\left(B_{j}\right)$ and $\varphi_{j}^{*}=\varphi_{j}^{*}\left(A_{j}\right)$. We have restricted the action $I$ to the sets $S_{1}$ and $S_{2}$ to form $J\left(B_{1}, B_{2}\right)$ and $G\left(A_{1}, A_{2}\right)$. Each of these two restricted forms of the action is stationary at the critical point $\left(u_{j}, \varphi_{j}\right)$, and because of the way they are constructed, the stationary results are said to be dual [1]. In deriving (19), the existence of $f_{j}^{-1}$ is assumed. It can be easily shown that

$$
G\left(A_{1}, A_{2}\right) \leqslant I\left(u_{1}, u_{2}, \varphi_{1}, \varphi_{2}\right) \leqslant J\left(B_{1}, B_{2}\right)
$$

if $W_{j}\left(u_{j}, \varphi_{j}\right)$ is convex in $u_{j}$, concave in $\varphi_{j}$ and at least one of these is definite.

It is, from equation (9), evident that $W_{j}\left(u_{j}, \varphi_{j}\right)$ is convex in $u_{j}$ and is definite. The sufficient condition for $W_{j}\left(u_{j}, \varphi_{j}\right)$ to be concave in $\varphi_{j}$ is that

$$
-d f_{j} / d \varphi_{j} \geqslant \gamma_{j} \geqslant 0 \text {. }
$$

Since the exact solution $\left(u_{j}, \varphi_{j}\right)$ is related through the Eqs. (9) and (11) we can take

$$
A_{j}=k_{j} T_{j} \psi_{j}
$$

where $\psi_{j}$ is any function satisfying the constraint

$$
\sigma_{1}^{*} k_{1} T_{1} \psi_{1}-\sigma_{2}^{*} k_{2} T_{2} \psi_{2}=h \text { on } \Gamma \text {. }
$$

Consequently (20) becomes

$$
G\left(k_{1} T_{1} \psi_{1}, k_{2} T_{2} \psi_{2}\right)=H\left(\psi_{1}, \psi_{2}\right) \leqslant I\left(u_{1}, u_{2}, \varphi_{1}, \varphi_{2}\right) \leqslant J\left(B_{1}, B_{2}\right)
$$

and $\psi_{j}=B_{j}$ will be the exact solution when the equality signs hold. From (22)

$$
\begin{aligned}
J\left(B_{1}, B_{2}\right)-H\left(\psi_{1}, \psi_{2}\right) & \geqslant J\left(B_{1}, B_{2}\right)-I\left(u_{1}, u_{2}, \varphi_{1}, \varphi_{2}\right) \\
& =\sum_{j=1}^{2}\left\{\left(T_{j} \xi_{j}, k_{j} T_{j} \xi_{j}\right)+\left\langle\xi_{j},-d f_{j}\left(\varphi_{j t}\right) \xi_{j} / d \varphi_{j}\right\rangle\right\},
\end{aligned}
$$

where $\xi_{j}=B_{j}-\varphi_{j}$ and $\varphi_{j t}=t B_{j}+(1-t) \phi_{j}, 0 \leqslant t \leqslant 1$. 
From (5), (6), and (23) it follows that

$$
\begin{aligned}
2\left[J\left(B_{1}, B_{2}\right)-H\left(\psi_{1}, \psi_{2}\right)\right] \geqslant & \sum_{j=1}^{2}\left\{\left\langle T_{j}^{*} k_{j} T_{j} \xi_{j}, \xi_{j}\right\rangle+\left\langle\xi_{j},-d f_{j}\left(\varphi_{j t}\right) \xi_{j} / d \varphi_{j}\right\rangle\right\} \\
& +\left\langle\sigma_{1}^{*} k_{1} T_{1} \xi_{1}, \xi_{1}\right\rangle_{\Gamma_{1}}+\left\langle\sigma_{2}^{*} k_{2} T_{2} \xi_{2}, \xi_{2}\right\rangle_{\Gamma_{2}} \\
& +\left\langle\sigma_{1}^{*} k_{1} T_{1} \xi_{1}, \xi_{1}\right\rangle_{\Gamma}-\left\langle\sigma_{2}^{*} k_{2} T_{2} \xi_{2}, \xi_{2}\right\rangle_{\Gamma} \\
\geqslant & \sum_{j=1}^{2}\left(\Lambda+\gamma_{j}\right)\left\langle\xi_{j}, \xi_{j}\right\rangle,
\end{aligned}
$$

where $\Lambda$ is a lower bound to the least eigenvalue of

$$
T_{j}^{*} k_{j} T_{j} \theta_{j}=\lambda \theta_{j} \text { in } D_{j}
$$

with $\theta_{j}=0$ on $\Gamma_{j}$ and $\theta_{1}=\theta_{2}$ and $\sigma_{1}^{*} k_{1} T_{1} \theta_{1}=\sigma_{2}^{*} k_{2} T_{2} \theta_{2}$ on $\Gamma$. Hence the required error estimate is

$$
\|\xi\|_{L^{2}}^{2} \leqslant 2[J-H] /(\Lambda+\gamma)
$$

where $\gamma=\min _{j} \gamma_{j}$.

4. Application to steady-state heat conduction in a composite medium. Let $D$ be a region in the $(x, y)$ plane consisting of two parts $D_{1}$ and $D_{2}$. The plane which occupies the region $D_{j}$ is a homogeneous material of thermal conductivity $k_{j}$. We want to investigate typical boundary value problems in steady-state heat conduction for the composite medium $D$ with the source term $f_{j}\left(\varphi_{j}\right)$, the temperature being prescribed on the boundaries. One matching condition is obtained by assuming that no sources are created on the interface. The other condition is obtained by requiring that the temperature be continuous at the interface, which is reasonable on physical grounds when $D_{1}$ and $D_{2}$ are in intimate contact.

The governing differential equation is given by

$$
L_{j} \varphi_{j}=-\nabla \cdot\left(k_{j} \nabla \varphi_{j}\right)=f_{j}\left(\varphi_{j}\right) \text { in } D_{j},
$$

with $\varphi_{j}=\alpha_{j}$ on $\Gamma_{j}$ and the matching conditions $\varphi_{1}=\varphi_{2}$ on $\Gamma$ and $\hat{n} \cdot k_{1} \nabla \varphi_{1}=\hat{n} \cdot k_{2} \nabla \varphi_{2}$ on $\Gamma$, where $\hat{n}$ is the unit outward normal vector to $\Gamma$.

The bounding functionals $J$ and $G$, for $f_{1}\left(\varphi_{1}\right)=-a^{2} \varphi_{1}+f_{1}(x, y)$ and $f_{2}\left(\varphi_{2}\right)=-b^{2} \varphi_{2}$ $+g_{2}(x, y)$, defined by (18) and (19) are given by

$$
\begin{aligned}
2 J\left(B_{1}, B_{2}\right)= & \int_{D_{1}}\left\{k_{1}\left(\nabla B_{1}\right)^{2}+a^{2} B_{1}^{2}-2 f_{1}(x, y) B_{1}\right\} d x d y \\
& +\int_{D_{2}}\left\{k_{2}\left(\nabla B_{2}\right)^{2}+b^{2} B_{2}^{2}-2 g_{2}(x, y) B_{2}\right\} d x d y
\end{aligned}
$$


and

$$
\begin{aligned}
-2 H\left(\psi_{1}, \psi_{2}\right)= & a^{-2} \int_{D_{1}}\left\{\left(L_{1} \psi_{1}\right)^{2}+f_{1}^{2}(x, y)-2 f_{1}(x, y) L_{1} \psi_{1}\right\} d x d y \\
& +k_{1} \int_{D_{1}}\left(\nabla \psi_{1}\right)^{2} d x d y+k_{2} \int_{D_{2}}\left(\nabla \psi_{2}\right)^{2} d x d y \\
& +b^{-2} \int_{D_{2}}\left\{\left(L_{2} \psi_{2}\right)^{2}+g_{2}^{2}(x, y)-2 g_{2}(x, y) L_{2} \psi_{2}\right\} d x d y \\
& -2 \int_{\Gamma_{1}} k_{1} \frac{\partial \psi_{1}}{\partial n} d s-2 \int_{\Gamma_{2}} \alpha_{2} k_{2} \frac{\partial \psi_{2}}{\partial n} d s .
\end{aligned}
$$

In our further calculations we take

$$
\begin{aligned}
& D_{1}=\{(x, y) /-1<x<0,-1<y<1\}, D_{2}=\{(x, y) / 0<x<1,-1<y<1\}, \\
& \alpha_{i}=0, \quad f_{1}(x, y)=f_{11}(x) f_{12}(y), \quad \text { and } g_{2}(x, y)=g_{11}(x) g_{12}(y) .
\end{aligned}
$$

4.1. Minimization of $J$. Let us assume that the solution $B_{j}$ is of the product form

$$
B_{j}(x, y)=m_{j}(x) n(y) .
$$

By substituting (30) in (28) we see that $J$ depends upon $m_{j}$ and $n$. If we assume that $n$ is an a priori known function satisfying $n(-1)=n(1)=0$, then $J$, as a functional of $m_{j}$ [5], is given by

$$
\begin{aligned}
2 J\left(m_{1}, m_{2}\right)= & \int_{-1}^{0}\left\{A_{11}\left(\frac{d m_{1}}{d x}\right)^{2}+A_{15} m_{1}^{2}(x)+A_{14} f_{11}(x) m_{1}(x)\right\} d x \\
& +\int_{0}^{1}\left\{B_{11}\left(\frac{d m_{2}}{d x}\right)^{2}+B_{15} m_{2}^{2}(x)+B_{14} g_{11}(x) m_{2}(x)\right\} d x,
\end{aligned}
$$

where the constants depend upon the assumed form of $n(y)$ :

$$
\begin{aligned}
& A_{11}(n)=\int_{-1}^{1} k_{1} n^{2}(y) d y ; \quad B_{11}(n)=\int_{-1}^{1} k_{2} n^{2}(y) d y, \\
& A_{12}(n)=\int_{-1}^{1} k_{1}\left(\frac{d n}{d y}\right)^{2} ; \quad B_{12}(n)=\int_{-1}^{1} k_{2}\left(\frac{d n}{d y}\right)^{2} d y, \\
& A_{13}(n)=\int_{-1}^{1} a^{2} n^{2}(y) d y ; \quad B_{13}(n)=\int_{-1}^{1} b^{2} n^{2}(y) d y, \\
& A_{14}(n)=-2 \int_{-1}^{1} f_{12}(y) n(y) d y, \quad B_{14}(n)=-2 \int_{-1}^{1} g_{12}(y) n(y) d y, \\
& A_{15}(n)=A_{12}+A_{13}, \quad \text { and } \quad B_{15}=B_{12}+B_{13} .
\end{aligned}
$$

We may determine $m_{j}$ so that $J$ is minimized. This leads to the following Euler-Lagrange equations for $m_{j}$ :

$$
\begin{array}{ll}
\frac{d^{2} m_{1}}{d x^{2}}-\eta_{1}^{2} m_{1}=\frac{A_{14}}{2 A_{11}} f_{11}(x), & -1<x<0, \\
\frac{d^{2} m_{2}}{d x^{2}}-\eta_{2}^{2} m_{2}=\frac{B_{14}}{2 B_{11}} g_{11}(x), & 0<x<1,
\end{array}
$$


with the conditions

$$
m_{1}(-1)=m_{2}(1)=0, \quad m_{1}(0)=m_{2}(0)
$$

and

$$
k_{1} \frac{d m_{1}}{d x}=k_{2} \frac{d m_{2}}{d x} \quad \text { at } x=0,
$$

where $\eta_{1}^{2}=A_{15} / A_{11}$ and $\eta_{2}^{2}=B_{15} / B_{11}$.

Maximization of $H$. Choose the trial function

$$
\psi_{j}(x, y)=\eta B_{j}(x, y),
$$

where $\eta$ is the unknown parameter to be determined. Substituting (34) in (29) we have

$$
-2 H\left(\psi_{1}, \psi_{2}\right)=\eta^{2}\left[I_{1}+I_{2}\right]-2 \eta I_{3}+I_{4},
$$

where

$$
\begin{aligned}
& I_{1}=\bar{a}^{2} \int_{D_{1}}\left(L_{1} B_{1}\right)^{2} d x d y+k_{1} \int_{D_{1}}\left(\nabla B_{1}\right)^{2} d x d y \\
& I_{2}=\bar{b}^{2} \int_{D_{2}}\left(L_{2} B_{2}\right)^{2} d x d y+k_{2} \int_{D_{2}}\left(\nabla B_{2}\right)^{2} d x d y \\
& I_{3}=\bar{a}^{2} \int_{D_{1}} f_{1}(x, y) L_{1} B_{1} d x d y+\bar{b}^{2} \int_{D_{2}} g_{2}(x, y) L_{2} B_{2} d x d y \\
& I_{4}=\bar{a}^{2} \int_{D_{1}} f_{1}^{2}(x, y) d x d y+\bar{b}^{2} \int_{D_{2}} g_{2}^{2}(x, y) d x d y .
\end{aligned}
$$

$d H / d \eta=0$ leads to $\eta=I_{3} / I_{1}+I_{2}$.

4.3. Numerical results. Numerical calculations are carried out with $k_{1}=0.18, k_{2}=0.14$, $f_{1}(x, y)=g_{2}(x, y)=x$ and $a^{2}=b^{2}=1$. The solution of (32) and (33) and the value of $\eta$ for

$$
n(y)=\frac{\cosh \mu y}{\cosh \mu}-1, \quad \mu=3.1379909,
$$

are given by $m_{j}(x)=p_{j} \exp \left[\eta_{j} x\right]+q_{j} \exp \left[-\eta_{j} x\right]+c_{j} x$ in $[-1,0]$ and $[0,1]$, respectively, where

$$
\begin{array}{lll}
p_{1}=0.323063133, & q_{1}=-0.047463513, & p_{2}=0.03837185 \\
q_{2}=0.23722777, & \eta_{1}=2.906658224, & \eta_{2}=3.167958905 \\
c_{1}=-0.850763427, & c_{2}=-0.920835684, & \eta=0.9379559949 .
\end{array}
$$

The corresponding extremum values of the functionals are found to be

$$
J=-0.09806582, \quad G=-0.20685117 \text {. }
$$

The lower bound to the least eigenvalue to the corresponding problem is taken to be [6] $\Lambda=0.6908723$.

Consequently, the mean square error is given by

$$
\|\xi\|_{L^{2}}^{2} \leqslant 0.1286735
$$


and one can improve the estimate by applying a more sophisticated method like the Finite Element Method.

5. Construction of Green's function. As an example of (1) and (2) with source or sink on the interface, we consider the one-dimensional heat conduction equation. If there is a unit sink at the origin and the boundary is kept at zero temperature, the one-dimensional heat conduction equation is given by [2]

$$
d^{2} \varphi / d x^{2}=\delta(x), \quad-1<x<1,
$$

with $\varphi(-1)=0, \varphi(1)=0$. Note that

$$
\langle\delta(x), \varphi(x)\rangle=\lim _{\varepsilon \rightarrow 0} \frac{1}{2}[\langle\delta(x-\varepsilon), \varphi(x)\rangle+\langle\delta(x+\varepsilon), \varphi(x)\rangle] .
$$

If $\varphi=\varphi_{j}, j=1,2,3$ in $[-1,-\varepsilon],[-\varepsilon, \varepsilon]$ and $[\varepsilon, 1]$, respectively, $(35)$ reduces to $d^{2} \varphi_{j} / d x^{2}=0$, $j=1,2,3$, in $(-1,-\varepsilon),(-\varepsilon, \varepsilon)$ and $(\varepsilon, 1)$, respectively, with the constraints

$$
\begin{aligned}
\frac{d \varphi_{2}}{d x}(-\varepsilon)-\frac{d \varphi_{1}}{d x}(-\varepsilon) & =\frac{1}{2} ; \quad \frac{d \varphi_{3}}{d x}(\varepsilon)-\frac{d \varphi_{2}}{d x}(\varepsilon)=\frac{1}{2} ; \\
\varphi_{1}(-\varepsilon) & =\varphi_{2}(-\varepsilon) ; \quad \varphi_{3}(\varepsilon)=\varphi_{2}(\varepsilon) ; \quad \varphi_{1}(-1)=\varphi_{3}(1)=0 .
\end{aligned}
$$

Application of the dual extremum principles leads to

$$
\varphi_{1}(x)=-\frac{1}{2}(x+1), \quad-1 \leqslant x \leqslant-\varepsilon ; \quad \varphi_{2}(x)=\frac{1}{2}(\varepsilon-1), \quad-\varepsilon \leqslant x \leqslant \varepsilon,
$$

and

$$
\varphi_{3}(x)=\frac{1}{2}(x-1), \quad \varepsilon \leqslant x \leqslant 1
$$

which is the exact solution when $\varepsilon \rightarrow 0$.

6. Concluding remarks. Dual extremum principles can be formulated for nonconvex and multiply-connected domains by subdividing the domain into convex domains and using the matching conditions on the cuts. Green's function, in two dimensions for the heat conduction equation, can be constructed by just extending the principle used in the case of one dimension.

\section{REFERENCES}

[1] A. M. Arthurs, Complementary variational principles, Oxford University Press, 1980

[2] I. Stakgold, Green's functions and boundary value problems, John Wiley and Sons, 1979

[3] R. E. Collin, Field theory of guided waves, McGraw Hill, New York, 1969

[4] (3. K. Batchelor, An introduction to fluid dynamics, Cambridge University Press, 1970

[5] L. V. Kantorovich and V. I. Krylov, Approximate methods of higher analysis, P. Noordhoff, 1958

[6] R. Courant and D. Hilbert, Methods of mathematical physics, Vol. 1, Wiley Eastern Pvt. Ltd., New Delhi, 1975 